\title{
GASIFICATION OF COAL-DERIVED CHARS IN SYNTHESIS GAS MIXTURES UNDER INTRAPARTICLE MASS- TRANSFER-CONTROLLED CONDITIONS
}

\author{
A. BLIEK, ${ }^{\dagger}$ J. C. LONT and W. P. M. VAN SWAAIJ \\ Department of Chemical Engineering. Twente University of Technology, P.O. Box 217, 7500 AE Enschede, \\ The Netherlands
}

(Received 5 April 1985)

\begin{abstract}
A model has been formulated to describe the quasi-steady-state gasification of coal-derived chars in gas mixtures where both the reactants carbon dioxide and steam, and the gasification products carbon monoxide and hydrogen are present. As such, these conditions reflect the situation found in most practical gasification systems.

The model presented is applied under conditions where intraparticle mass transfer is rate-controlling. Intraparticle heat transfer is neglected. In view of the non-equimolar gasification reactions, the mass fux equations are derived from the continuum limit of the dusty gas model. These flux equations are combined with strongly non-linear Langmuir-Hinshel wood kinetics for the gasification reactions. The model accounts for local variations of the diffusive and convective permeability parameters, as well as variations in the reactive surface area, during burnoff of a char particle.

The impact of the various relevant mass-transfer parameters, the gasification temperature and pressure, and the char particle size on the gasification behaviour is discussed. A comparison is made between the present model and existing models, and various modelling approaches are critically reviewed.
\end{abstract}

\section{INTRODUCTION}

An important aspect of the mathematical modelling of coal gasification reactors is the description of the comparatively slow reaction of coal-derived chars with carbon dioxide and steam. Especially in the case of fixed and fluidized bed reactors, the operating conditions are generally such that the rate-controlling step in this reaction is formed by the intraparticle mass transfer of reactants and reaction products. As will be shown elsewhere for fixed bed gasifiers [1], the overall gasification rate of chars in carbon dioxide and steam may influence the design of practical gasification reactors.

Whereas the subject of kinetically controlled gasification has received much attention, comparatively little is known about the role of intraparticle mass transfer and the role of the char pore structure. In particular, this applies to those cases where more than one oxidant is present. Mass-transfer-controlled gasification of chars is sometimes modelled along similar lines as the reaction and diffusion in catalyst particles. However, some marked differences exist. The pore structure of chars is distinctly different from that found for most catalyst particles. In addition, the char is consumed in the course of gasification and the pore structure changes significantly. It is well known that the counteracting phenomena of pore growth and pore overlap can give rise to substantial variations of the reactive surface area during burnout. As was shown recently by Srinivas and Amundson [2], this factor cannot be neglected. During conversion of a char

\footnotetext{
${ }^{\dagger}$ Present address: Dutch Philips Companies-Glass, HBU, 5600 MD Eindhoven, The Netherlands.
}

particle, the porosity increases. This has a dramatic influence on the mass transport parameters. Desai and Yang [3] demonstrated that the use of a constant effective diffusivity may lead to large errors in the predicted conversion-time behaviour of a char particle. Therefore conversion-dependent effective transport parameters should be used.

In the case of catalyst particles, it is usually assumed that concentration gradients can develop only in the direction of macroscopic flow. The flux equations are thus solved with the intraparticle radial distance as the characteristic length-scale. For all pores, located at the same macroscopic position within the particle, the reaction conditions are thus assumed to be uniform. This "smooth-field" assumption [4] may be valid for coarsely porous particles, with a highly interlinked pore system. In contrast, coal chars are known to have a wide pore size distribution. Moreover, most of the reactive surface area is concentrated in the micropore regime and therefore heteroporosity effects may be important. The question of pore interconnectivity was treated explicitly by Simons and Finson [5] and Gavalas [6]. On the basis of the pore tree model, presented by the former, the validity of the smooth field approach will be discussed.

Both Hashimoto and Silveston [7] and Wen and Wu [8] have analysed the mass-transfer-controlled gasification of chars by a single oxidant. The presence of reaction products was neglected and therefore the treatment could be simplified to the calculation of particle effectiveness factors on the basis of the Thiele modulus. It is well known that the rate of char gasification in carbon dioxide or steam can be influenced strongly by the presence of the reaction products carbon monoxide and hydrogen. If these 
species are present, the diffusion of both products and reactants must be considered. Even for the case of a single oxidant and a single reaction product, only approximate solutions for the Thiele modulus are known [9]. In the case of char gasification in synthesis gas mixtures, various reactions proceed simultaneously and analytical approximations on the basis of the Thiele modulus are not available. Therefore the solutions must be obtained by numerical analysis.

A further complication arises from the fact that gasification reactions are accompanied by a net mole flux, or, better, a net momentum flux. Hence, intraparticle pressure gradients will develop. For dilute systems, or when the molecular weights of all flowing species are approximately equal, the component fluxes are given by the Stefan-Maxwell equations. These equations were applied by Yang and Stcinberg $[10]$ to describe mass-transfer-controlled gasification of graphite in $\mathrm{CO}-\mathrm{CO}_{2}-\mathrm{Ar}$ mixtures and by Arri and Amundson [11] to describe ash layer diffusion-controlled gasification of chars in a $\mathbf{H}_{2} \mathrm{O}-\mathrm{H}_{2}$ environment. In a later treatment, Srinivas and Amundson [2] used the Stefan-Maxwell fiux cquations for the gasification of chars in $\mathrm{CO}-\mathrm{CO}_{2}-\mathrm{H}_{2}-\mathrm{H}_{2} \mathrm{O}-\mathrm{CH}_{4}$. In the present model the dusty gas flux equations [12] will be applied, as these are, at least fundamentally, more correct than the Stefan-Maxwell equations.

As is illustrated above, the mathematical modelling of mass-transfer-controlled gasification of chars in synthesis gas mixtures involves multicomponent mass transfer under the combined action of pressure and composition gradients, non-linear kinetics due to product inhibition and both a varying internal surface area and varying mass-transfer parameters during char burnoff.

\section{MODEL FORMULATION}

\subsection{General assumptions}

The model outlined below describes the masstransfer-controlled gasification of a single char particle in a gaseous environment consisting of a mixture of $\mathrm{CO}, \mathrm{CO}_{2}, \mathrm{H}_{2} \mathrm{O}, \mathrm{H}_{2}$ and $\mathrm{N}_{2}$, as found in air-blown gasifiers. The gasification process is assumed to attain a quasi-steady state and the ambient conditions of the particle are fixed in time. It will be shown that the intraparticle temperature gradients are small and therefore the particle is assumed to be isothermal.

The model is applied to two coal-derived chars with distinctly different structural parameters and intrinsic reactivities. The impact of the main imposable variables on the particle effectiveness factor will be discussed and the intraparticle conditions for various cases will be presented.

\subsection{Kinetics of gasification}

The gasification of chars in synthesis gas mixtures proceeds through the reaction of carbon active sites with carbon dioxide and steam. In the case of highpressure gasification, the direct hydrogenation of surface methylene groups to methane may also have to be included. In our model this reaction will be ignored. Along with two heterogeneous reactions, the homogeneous shift reaction is also accounted for:

$$
\begin{gathered}
\mathrm{C}+\mathrm{CO}_{2} \stackrel{\mathrm{R}_{1}}{\rightarrow} 2 \mathrm{CO} \\
\mathrm{C}+\mathrm{H}_{2} \mathrm{O} \stackrel{\mathrm{R}_{1 \mathrm{I}}}{\rightarrow} \mathrm{CO}+\mathrm{H}_{2} \\
\mathrm{CO}+\mathrm{H}_{2} \mathrm{O} \underset{\mathrm{s}, i}{\stackrel{k_{\mathrm{s}, j}}{\rightleftharpoons}} \mathrm{CO}_{2}+\mathrm{H}_{2} .
\end{gathered}
$$

Under conditions remote from equilibrium the intrinsic heterogeneous reaction rates are given by Langmuir-Hinshelwood equations:

$$
\begin{aligned}
R_{\mathrm{I}} & =\frac{K_{1} \mathrm{PCO}_{2}}{1+K_{2} \mathrm{PCO}_{2}+K_{3} P C O} \quad\left(\mathrm{~mol} \mathrm{~m}^{-2} \mathrm{~s}^{-1}\right) \\
R_{\mathrm{II}} & =\frac{K_{4} \mathrm{PH}_{2} \mathrm{O}}{1+K_{5} \mathrm{PH}_{2} \mathrm{O}+K_{6} \mathrm{PH}_{2}} \quad\left(\mathrm{~mol} \mathrm{~m}^{-2} \mathrm{~s}^{-1}\right)
\end{aligned}
$$

The rate and equilibrium constants for the two chars presently investigated have been reported elsewhere [1] and are listed in Table 2. It was also shown that the overall carbon conversion rate can be expressed in good approximation as:

$$
\boldsymbol{R}=\boldsymbol{R}_{\mathbf{I}}+\boldsymbol{R}_{\mathrm{II}} \text {. }
$$

The homogeneous shift reactions are assumed to be infinitely fast so that equilibrium is attained anywhere in the char particle [13]:

$$
K_{\mathrm{s}}=\frac{k_{\mathrm{s}, j}}{k_{\mathrm{s}, i}}=\frac{\mathrm{PCO}_{2} \times \mathrm{PH}_{2}}{\mathrm{PH}_{2} \mathrm{O} \times \mathrm{PCO}}
$$

with

$$
\log _{10} K_{\mathrm{s}}=-1.6945+1855.6 / T \text {. }
$$

The development of the internal surface area of a char particle during burnoff is governed mainly by its initial pore structure. For coal-derived chars it is often observed that the specific surface area per unit of particle volume attains a maximum as a function of the burnoff. This can be attributed to the opposing effects of pore growth and pore overlap. For solids with a pore size distribution, both Hashimoto and Silveston [7] and Bhatia and Perlmutter [14] have presented pore growth models which account for these phenomena. The comparatively simple model equations of Bhatia and Perlmutter were found to be in satisfactory agreement with experimental data for the two chars investigated [1]:

$$
S_{p}=S_{p}^{\circ}\left[1-\psi \ln \left(1-X_{c}\right)\right]^{1 / 2} .
$$

The initial char porosity $\varepsilon_{p}^{\circ}$, the initial internal surface area $S_{\mathrm{p}}^{\circ}$ and the surface area development parameter $\psi$ are listed in Table 2.

\subsection{A pore model for chars}

It is well known that coal-derived chars have a wide pore size distribution, ranging from super-micropores to microcracks [15]. As the active surface area is largely concentrated in the micropore regime, it is 
Table 1. Analysis of the coals investigated

\begin{tabular}{|c|c|c|}
\hline $\begin{array}{l}\text { Coal rank: } \\
\text { Denomination: }\end{array}$ & $\begin{array}{c}\text { Semi- } \\
\text { anthracite } \\
\mathbf{A}\end{array}$ & $\begin{array}{c}\text { HVB } \\
\text { bituminous } \\
\mathrm{B}\end{array}$ \\
\hline $\begin{array}{ll}\begin{array}{l}\text { Proximate analysis } \\
\text { volatile matter }(\% \text { d.a.f. }) \\
\text { ash }\end{array} & (\% \text { dry }) \\
\text { fixed carbon } & (\% \text { d.a.f. }) \\
\text { moisture } & (\% \text { a.r. })\end{array}$ & $\begin{array}{r}13.1 \\
8.4 \\
86.9 \\
3.0\end{array}$ & $\begin{array}{r}35.7 \\
6.3 \\
64.3 \\
6.0\end{array}$ \\
\hline 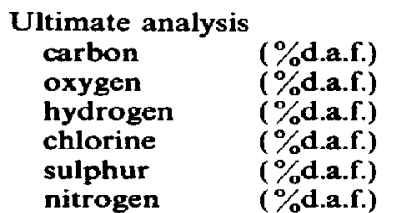 & $\begin{array}{l}90.2 \\
3.3 \\
4.0 \\
0.11 \\
0.8 \\
1.0\end{array}$ & $\begin{array}{r}81.8 \\
11.6 \\
5.1 \\
0.14 \\
0.45 \\
0.58\end{array}$ \\
\hline
\end{tabular}

relevant to establish how macro- and micropores are interconnected. In the case of porous solids with a bimodal pore size distribution, it is often assumed that micropores radiate from macropores (see, for instance, [16]). Here, a similar approach is taken. It will be assumed that macropores act as the main carriers of reactants and reaction products, whereas their contribution to the heterogeneous reactions is negligible. On the other hand, the micro- and mesopores are assumed to radiate from these feeder pores. Hence, micro-/ mesopores play no role in macroscopic transport and only act as a source of reaction products and as a sink of reactants. On the scale of a particle, the pore structure can be considered homogeneous, with randomly located feeder pores and a homogeneously distributed reactive surface area. Hence, the flux parameters for macroscopic transport are not influenced by the particle size, as long as the particle size does not approach the mean size of the feeder pores. On the scale of a macropore, the pores are not necessarily distributed in a random fashion.
In this respect opposing views are taken by Gavalas [6] and Simons and Finson [5]. In both treatments the question of pore interconnectivity is discussed. Gavalas concludes that small pores end up mainly in larger ones, through two intersections. In contrast to this, Simons and Finson conclude that all pores end up in larger ones through a single intersection. According to these authors, the pore system would therefore resemble a tree-like system. Because all pores ultimately end up in the solid structure, the tree model implies that char particles are impermeable. Obviously this cannot be true, unless in the limit of extremely small particles. For larger particles, some pores must be well crosslinked with others. In our view, this applies mainly to the system of macropores. Therefore the pore tree model will be used here as an extreme case with respect to pore interconnectivity, valid only for the micro-/mesopore system. The maximum size of a tree-like pore system is governed by constraints with regard to the pore wall area of the tree, which must obviously be smaller than the specific surface area of the whole particle. Using the relations presented by Simons and Finson [5], it is easily shown that the pore tree system cannot apply for pores larger than $0.2-0.4 \mu \mathrm{m}$. The point to be made here is that for this range of maximum pore tree size, the pore tree effectiveness factor is close to unity for temperature levels up to $1400^{\circ} \mathrm{C}$ (see Appendix A). Consequently we need not concern ourselves with the way in which the micro-/mesopore system is connected to the feeder pore system, and the smooth-field approach may be assumed valid.

In the present treatment the char pore structure is assumed to consist of large feeder pores, from which smaller pore branches radiate (Fig. 1). The existence of "bottle-neck" pores is ignored and the feeder pore system is assumed to be well interlinked and homogeneously distributed. As will be shown in the next section, diffusion in the major part of the feeder pore

Table 2. Pores structure data and kinetics of gasification

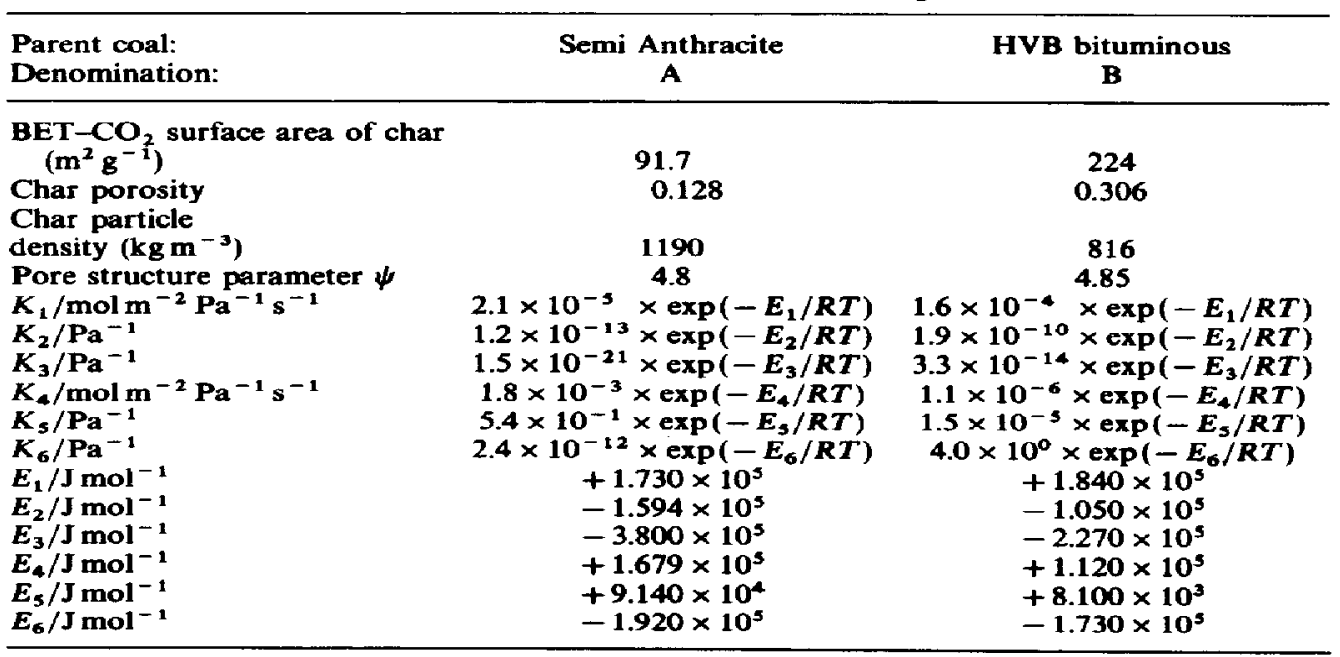




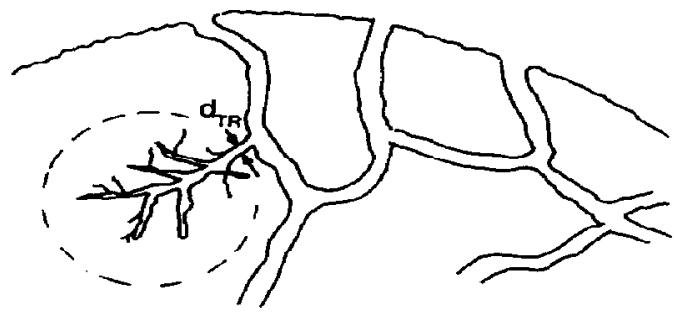

Fig. 1. Schematic representation of the pore structure of chars.

system is in the continuum regime. For this reason the overall diffusivity is not affected much by the pore size distribution. In other words, the feeder pore system can be considered homoporous, which allows us to use the dusty gas model, with empirically derived diffusive and viscous permeabilities.

\subsection{Intraparticle mass transfer}

2.4.1. Flux equations. The formulation of the multicomponent mass flux equations for the char feeder pore system is hampered by the fact that both composition and pressure gradients exist. According to Graham's law for isobaric diffusion, generalized to a multicomponent mixture [17], intraparticle pressure gradients will develop whenever the following inequality is satisfied:

$$
\sum_{i=1}^{n}\left[N_{i} M_{i}^{1 / 2}\right] \neq 0
$$

In fact, inequality (7) states that a net transfer of momentum must exist in order to bring about intraparticle pressure differences. This condition is met in the case of char gasification, since in this process a net transfer of carbon out of the particle exists. For this reason the flux equations are derived from the dusty gas model, which was formulated in a suitable compact form by Mason et al. [12] following previous work of Deriaguin and Bakanov [18].

The general dusty gas fiux equations for isothermal particles are formulated as:

$$
\begin{aligned}
\frac{N_{i}}{D_{\mathrm{k}, i}^{\mathrm{e}}}+\sum_{\substack{j=1 \\
j \neq 1}}^{n} \frac{\left[X_{j} N_{i}-X_{i} N_{j}\right]}{D_{i, j}^{e}}= \\
\quad-\frac{P}{R T} \nabla X_{i}-\left\{\frac{B_{0} P}{\mu_{\mathrm{m}} D_{\mathrm{k}, i}^{e}}+1\right\} \frac{X_{i}}{R T} \nabla P .
\end{aligned}
$$

For various limiting cases eq. (8) reduces to simpler forms, which have appeared from earlier work, for instance on isobaric transition regime diffusion [19-22]. The dusty gas equation contains three transport parameters, namely the continuum diffusivity $D_{i, j}^{e}$, the Knudsen diffusivity $D_{k, i}^{c}$ and the viscous permeability $B_{0}$. For a single pore, with diameter $d_{p o}$, these parameters are defined by:

$$
\begin{aligned}
B_{\mathrm{o}} & =d_{\mathrm{po}}^{2} / 32 & \left(\mathrm{~m}^{2}\right) \\
D_{\mathrm{k}, i} & =1 / 3 d_{\mathrm{po}}\left\{\frac{8 R T}{M_{i} \pi}\right\}^{1 / 2} & \left(\mathrm{~m}^{2} \mathrm{~s}^{-1}\right)
\end{aligned}
$$

$$
D_{i, j}=C_{2} \frac{T^{1.5}}{P \sigma_{i, j}^{2} \Omega_{\mathrm{d}}}\left\{\frac{1}{M_{i}}+\frac{1}{M_{j}}\right\}^{1 / 2} \quad\left(\mathrm{~m}^{2} \mathrm{~s}^{-1}\right)
$$

with $C_{2}=1.86 \times 10^{-22} \mathrm{~N} \mathrm{~m}^{2} \mathrm{~kg}^{1 / 2} \mathrm{~mol}^{-1 / 2} \mathrm{~K}^{-1.5}$ $s^{-1}$.

For a binary $\mathrm{CO}_{2}-\mathrm{N}_{2}$ mixture it is easily shown on the basis of eqs (9)-(11) that $B \mathrm{o} P / \mu_{\mathrm{m}}>D_{k, i}>D_{i, j}$ for pores larger than $2 \mu \mathrm{m}$. For the largest part of the feeder pore system in coal-derived chars this holds, and consequently the dusty gas model can be simplified to its continuum limit:

$$
\begin{array}{r}
\sum_{\substack{j=1 \\
j \neq i} \frac{\left[X_{j} N_{i}-X_{i} N_{j}\right]}{D_{i, j}^{e}}}=-\frac{P}{R T} \nabla X_{i}-\frac{X_{i}}{R T} \\
\times\left\{1-\frac{1}{D_{\mathrm{k}, i}^{\mathrm{e}} \sum_{j=1}^{n}\left(X_{j} / D_{\mathrm{k}, j}^{\mathrm{e}}\right)}\right\} \nabla P .
\end{array}
$$

For an $n$-component mixture, only $(n-1)$ equations of the type of eq. (12) are independent. The set is completed by the pressure equation (13):

$$
\sum_{j=1}^{n}\left(\frac{N_{j}}{D_{\mathrm{k}, j}^{e}}\right)=-\frac{B_{\mathrm{o}} P}{\mu_{\mathrm{m}} R T} \sum_{j=1}^{n}\left(X_{j} / D_{\mathrm{k}, j}^{\mathrm{c}}\right) \nabla P \text {. }
$$

Equation (12) reduces to the conventional Stefan-Maxwell equations only for $D_{\mathbf{k}, j}^{\mathbf{e}}=D_{\mathbf{k}, i}^{\mathrm{e}}$ for any $i, j$. This comes down to the assumption of equal molecular weights for all components. In that case eq. (13) reduces to the D'Arcy law.

The application of the dusty gas model to coalderived chars has recently been criticized by $Y$ ang and Liu [23]. As was correctly stated by these authors, the dusty gas model was derived under the assumption of homogeneously distributed dust particles. When the model is applied to porous solids, the solid structure must in fact be homoporous. For heteroporous solids, such as chars, corrections must be made $[24,25]$. At present, the dusty gas equations are applied to the macropores, which can be assumed homoporous, as discussed in Section 2.3. Therefore the application of the dusty gas model will not result in significant crrors.

2.4.2. Mass-transfer parameters. The transport parameters $D_{\mathbf{k}, j}^{\mathrm{c}}$ and $D_{i, j}^{\mathrm{c}}$ can be expressed in terms of solid permeabilities:

$$
\begin{aligned}
& D_{i, j}^{\mathrm{e}}=k_{\mathrm{o}} D_{i, j} \\
& D_{\mathrm{k}, j}^{\mathrm{e}}=k_{1}\left\{\frac{8 R T}{\pi M_{j}}\right\}^{1 / 2} .
\end{aligned}
$$

The permeability factors $k_{0}, k_{1}$ and $B_{0}$ are a function of the pore structure only. As was shown by Feng et al. [26] and by Chen and Rinker [25], these permeability constants can be related to the internal surface area and pore size distribution of a solid. However, it is always necessary to introduce simplifying assumptions, for instance with regard to the pore shape or the existence of dead-end pores. Also, in the case of char gasification, the pore structure changes rapidly with conversion. Therefore we prefer to use empirical relations, which account for the conversion of the 
solid. Because the dusty gas model is applied in its continuum limit, the Knudsen diffusivity need not be considered. It was shown elsewhere [1] that the viscous and continuum diffusion permeabilities of coal chars can be related to the particle porosity $\varepsilon_{\mathrm{p}}$ by empirical equations:

$$
\begin{aligned}
& B_{\mathrm{o}}=4 \times 10^{-11} \frac{\left(\varepsilon_{\mathrm{p}}\right)^{3}}{\left(1-\varepsilon_{\mathrm{p}}\right)^{2}} \quad\left(\mathrm{~m}^{2}\right) \\
& k_{\mathrm{o}}=\frac{1}{\tau} \epsilon_{\mathrm{p}}^{\beta}
\end{aligned}
$$

with $\beta=3$ and $\tau=2.5$.

Equation (17) is commonly used for coal-derived chars, with varying values of the tortuosity factor $\tau$ and $\beta[2,3,8,27-29]$.

Usually very low values of the diffusive permeability $k_{\mathrm{o}}$ are found, especially for low-porous chars. One may therefore speculate that the experimentally obtained diffusivities may in fact represent transition regime diffusivities. Diffusion measurements in coal chars are usually carried out at room temperature. Whereas at this temperature the diffusion may be in the continuum regime, the diffusion may shift to the transition regime at higher temperature levels. This is caused by the different temperature coefficients for $D_{i, j}$ and $D_{k, j}$ [see eqs (10) and (11)]. This matter was recently discussed by Yang and Liu [23], who, in contrast to nearly all other investigators, obtained effective diffusivities for graphite at high temperatures. These authors showed that the diffusivity is proportional to $T^{1.10-1.17}$ for $T=295-978 \mathrm{~K}$. This result suggests transition regime diffusion, which would be contrary to our view that transit diffusion in coal chars is largely in the continuum diffusion regime. However, it was shown in a subsequent paper by Desai and Yang [3] that the effoctive diffusivity in graphite is proportional to $T^{1.05}$ even at $T=1873 \mathrm{~K}$. Clearly the two results are conflicting, since in the case of true transition regime diffusion the temperature dependence of the effective diffusivity should change considerably for different temperature regimes. Desai and Yang proposed eq. (17), with $\beta=2$ and $D_{i, j}$ replaced by the transition regime diffusivity $D_{t}$, according to the Bosanquet interpolation formula:

$$
\frac{1}{D_{i, i}}=\frac{1}{D_{i, j}}+\frac{1}{D_{k, i}}
$$

For the graphite investigated by Desai and Yang, with $\varepsilon_{p}=0.177$, and an assumed value of $\tau=2.5$, eq. (18) yields an overall temperature dependence of the effective diffusivity of $T^{1.05}$ at $1873 \mathrm{~K}$, only for a mean pore size of $\sim 5 \mu \mathrm{m}$. In this case eqs (17) and (18) yield an effective diffusivity of $2.7 \times 10^{-6} \mathrm{~m}^{2} \mathrm{~s}^{-1}$ at $1873 \mathrm{~K}$. If, instead, continuum diffusion control is assumed, eq. (17) with $\beta=3$ and $\tau=2.5$ results in an overall effective diffusivity of $8.5 \times 10^{-7} \mathrm{~m}^{2} \mathrm{~s}^{-1}$, which is much closer to the experimentally observed value of $1.6 \times 10^{-7} \mathrm{~m}^{2} \mathrm{~s}^{-1}$ [3]. In view of the inconsistencies discussed above, we therefore feel that the assumption of continuum diffusion control is reasonable, and that incorporation of Knudsen diffusion effects is not really justified. At higher burnoff levels this conclusion can be expected to hold even more.

\subsection{Conservation equations}

In the present model the effects of intraparticle heat transfer are ignored. This can be justified on the basis of the small intraparticle temperature gradients. In the case of a single oxidant, the maximum intraparticle temperature difference can be estimated from the Prater criterion for strongly diffusion controlled reactions [30]:

$$
\Delta T_{\max }=\frac{-\Delta H_{\mathrm{r}} D_{i, \mathrm{~m}}^{\mathrm{e}}}{\lambda_{\mathrm{s}}^{\mathrm{e}}} \frac{P X_{i}}{R T}
$$

For the carbon- $\mathrm{CO}_{2}$ reaction at $1400 \mathrm{~K}$, with $\lambda_{\mathrm{s}}^{\mathrm{e}}=2 \mathrm{~J} \mathrm{~m}^{-1} \mathrm{~K}^{-1} \mathrm{~s}^{-1}, X_{i}=0.5, P=0.1 \mathrm{MPa}, \Delta H_{\mathrm{r}}$ $=174 \mathrm{KJ} \mathrm{mol}^{-1}$ and $D_{i, \mathrm{~m}}^{\mathrm{e}}=10^{-5} \mathrm{~m}^{2} \mathrm{~s}^{-1}$, the maximum intraparticle temperature difference is estimated to be $4 \mathrm{~K}$. An exact value of $\Delta T$ for char gasification in synthesis gas mixtures can be obtained from the numerically calculated composition gradients. This showed that for the conditions investigated the maximum intraparticle temperature difference does not exceed $7 \mathrm{~K}$.

For the five-component mixture considered here, four independent flux equations are derived from eq. (12), together with $\Sigma X_{i}=1$. The intraparticle pressure is governed by eq. (13).

The appropriate mass conservation equations are formulated in their pseudo-stationary form, assuming the structural changes of the solid to be slow. Because the char particles are assumed to consist of carbon only, the net flux of all other elements must be zero anywhere in the particle. Hence, the following conservation equations apply:

$$
\begin{array}{ll}
\text { Oxygen balance : } & N_{\mathrm{CO}}+2 N_{\mathrm{CO}_{2}}+N_{\mathrm{H}_{2} \mathrm{O}}=0 \\
\text { Hydrogen balance : } & N_{\mathrm{H}_{2}}+N_{\mathrm{H}_{2} \mathrm{O}}=0 \\
\text { Nitrogen balance : } & N_{\mathrm{N}_{2}}=0 .
\end{array}
$$

The consumption of steam and carbon dioxide is governed by the heterogeneous reactions, according to eqs (1) and (2), as well as by the homogeneous shift reaction. As the homogeneous shift reaction is assumed to be at equilibrium anywhere in the particle, the conservation equations for steam and carbon dioxide are replaced by the conservation equation for carbon and the homogeneous shift equilibrium condition, eq. (4):

$$
\text { Carbon balance: } \frac{1}{r^{2}} \nabla\left\{r^{2}\left[N_{\mathrm{CO}}+N_{\mathrm{CO}}\right]\right\}=R_{\mathrm{v}} \text {. }
$$

The local volumetric reaction rate $R_{\mathrm{v}}$ depends on the intrinsic reactivity and on the local internal surface area:

$$
\begin{aligned}
R_{\mathrm{v}} & =\left\{R_{\mathrm{I}}+R_{\mathrm{II}}\right\} \rho_{\mathrm{o}} S_{\mathrm{p}}^{\circ}\left(1-X_{\mathrm{c}}\right)\left[1-\psi \ln \left(1-X_{\mathrm{c}}\right)\right]^{1 / 2} \\
& =-\frac{1}{12} \frac{1}{V_{\mathrm{p}}} \frac{\mathrm{d}}{\mathrm{d} t}\left(\rho_{\mathrm{s}} V_{\mathrm{p}}\right)
\end{aligned}
$$


It will be assumed here that the ash layer surrounding the char particle segregates when a critical char conversion of 0.94 is attained. This assumption governs the change in size of a particle during burnout.

The total set of equations describing the system investigated contains 11 parameters, namely five fluxes, five mole fractions and the pressure. Using the conservation equations (20)-(22), the shift equilibrium condition (4) and $\Sigma X_{i}=1$, five variables can be eliminated, for which we arbitrarily take the mole fluxes of hydrogen, steam and nitrogen, as well as the mole fractions of carbon dioxide and nitrogen. Using the five dusty gas flux equations (12) and (13), the mole fraction gradients for all components can be expressed as a function of the remaining variables $X_{\mathrm{H}_{2}}, \boldsymbol{X}_{\mathrm{H}_{2} \mathrm{O}}$, $X_{\mathrm{CO}}, N_{\mathrm{CO}}, N_{\mathrm{CO}_{2}}$ and $P$. Numerical calculations can now be started with known values of $X_{\mathrm{H}_{2} \mathrm{O}}, X_{\mathrm{H}_{2}}$ and $X_{\text {co }}$ in the gas film surrounding the particle. Initially $N_{\mathrm{CO}}$ and $N_{\mathrm{CO}_{2}}$ are guessed. The value of one of these parameters, for instance $N_{\mathrm{CO}_{2}}$, can be improved by iteration at each place-level, using the carbon balance (23). In this way, a forward finite difference scheme is started with only one unknown variable, in this case $N_{\text {Co. }}$. The initial estimate of $N_{\text {CO }}$ is improved by iteration over the whole particle, until the particle centre boundary conditions are satisfied.

\subsection{Boundary conditions}

The interphase mass transfer between the particle and the ambient gas is described on the basis of the film theory. Because a net mole flux out of the particle exists, the mass flux equations are formulated for simultaneous diffusive and convective flow, with corrected gas film mass-transfer coefficients [31]:

$$
\begin{aligned}
& N_{i}=X_{i}^{\mathrm{s}} \sum_{j=1}^{n} N_{j}+k_{\mathrm{g}, i}\left[X_{i}^{\mathrm{s}}-X_{i}^{\mathrm{b}}\right] \\
& k_{\mathrm{g} i}^{\bullet_{i}}=k_{\mathrm{g}, i} \frac{\gamma}{\mathrm{e}^{\gamma}-1}
\end{aligned}
$$

$\gamma=-\sum_{j=1}^{n}\left(N_{j} / k_{\mathrm{g}, j}\right)$ for a net flux into the particle $\gamma=+\sum_{j=1}^{n}\left(N_{j} / k_{\mathrm{g} j}\right)$ for a net flux out of the particle.

The mass-transfer coefficient at zero net mole flux, $k_{\mathrm{g}, \text {; }}$, is conventionally expressed in terms of the particle Sherwood number [32]:

$$
k_{\mathrm{g}, i}=\frac{D_{i, \mathrm{~m}}}{d_{\mathrm{p}}} S h \frac{P}{R T}
$$

and

$$
D_{i, \mathrm{~m}}=\frac{\left(1-X_{i}\right)}{\sum_{\substack{j=1 \\ j \neq i}}^{n}\left(X_{j} / D_{i, j}\right)}
$$

Boundary conditions are formulated with respect to the bulk of the gas phase and with respect to the particle centre:

$$
\begin{aligned}
& \text { Bulk conditions: } \quad X_{i}=X_{i}^{b} \text { for } \mathrm{CO}, \mathrm{H}_{2} \text { and } \mathrm{CO}_{2} \\
& \sum X_{i}=1 \\
& X_{\mathrm{H}_{2} \mathrm{O}}^{\mathrm{b}}=K_{\mathrm{s}}\left[\frac{X_{\mathrm{CO}_{2}} X_{\mathrm{H}_{2}}}{X_{\mathrm{CO}}}\right]^{b} \\
& \boldsymbol{P}=\boldsymbol{P}^{\mathbf{b}} \\
& \sum X_{i}=1 \\
& \nabla P=0 \text {. }
\end{aligned}
$$

Although the shift reaction equilibrium is assumed to be satisfied for the bulk of the gas phase as well as for the gas-solid interphase, it is not necessarily satisfied within the gas film because the gas film concentrations are governed by the diffusivities of the flowing species. Deviations from shift equilibrium in the gas film can be expected to be small, however, and this effect is ignored.

\section{RESULTS AND DISCUSSION}

The modelling equations were solved by numerical procedures, which will not be treated here. The gasification behaviour of a single char particle was analysed for a standard set of conditions, where only one parameter was varied at a time. It should be noted, however, that the gasification temperature and the bulk gas composition are coupled through the homogeneous shift equilibrium relation. Therefore these parameters cannot be varied independently. In the present case the bulk mole fraction of hydrogen and nitrogen were treated as the dependent variables. Hence, different gasification temperatures result in a corresponding change in the bulk hydrogen and nitrogen mole fraction.

The main variables governing the gasification behaviour of a single char particle are the particle size, the particle porosity and the gasification temperature. For the low-temperature regime, also the presence of hydrogen, which is a strong inhibitor, may affect the gasification rate.

Especially low-porous char particles rapidly enter the mass-transfer-controlled gasification regime when the temperature is raised. This is accompanied by the development of intraparticle mole fraction gradients for all species present. A typical example of the intraparticle gas composition is presented in Fig. 2. Steep gradients may exist in particular for $\mathrm{CO}$, because 1 mol of $\mathrm{CO}_{2}$ produces $2 \mathrm{~mol}$ of $\mathrm{CO}$. The homogeneous shift reaction may produce an additional amount of $\mathrm{CO}$. In comparison, the mole fraction gradient of hydrogen is quite small. This is a consequence of both the reaction stoichiometry, as $1 \mathrm{~mol}$ of $\mathrm{H}_{2} \mathrm{O}$ produces only $1 \mathrm{~mol}$ of $\mathrm{H}_{2}$, and of the high diffusivity of hydrogen. It is interesting to investigate the impact of taking an infinite binary diffusivity for hydrogen, as was proposed by Srinivas and Amundson [2]. A comparison between the particle effectiveness factor in the case of a finite hydrogen diffusivity and in the case of an infinite hydrogen diffusivity is presented in Fig. 3. The particle effectiveness factor is defined 


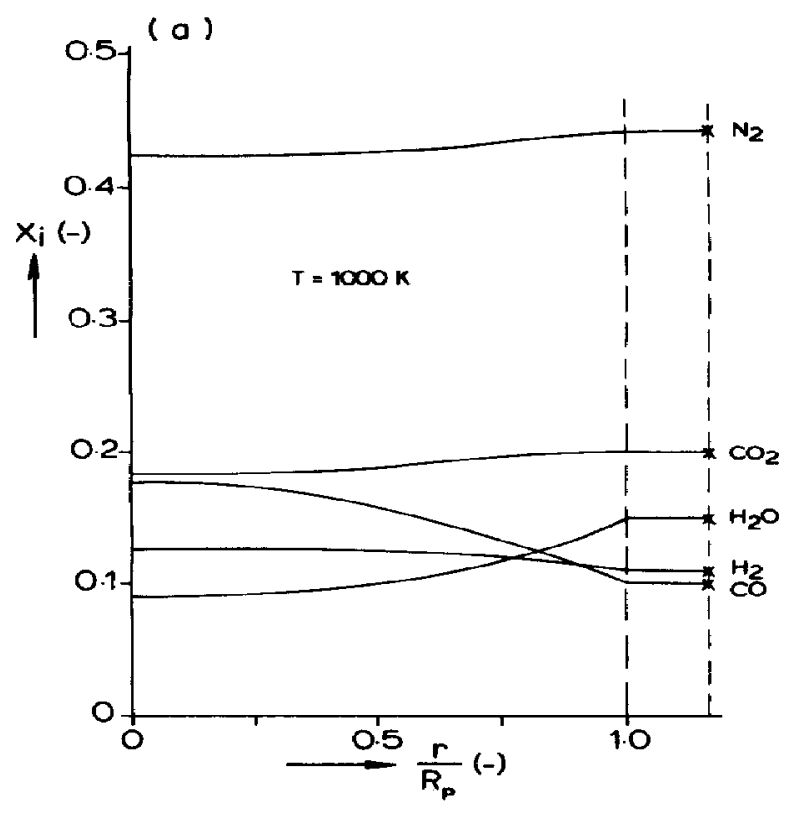

( b )
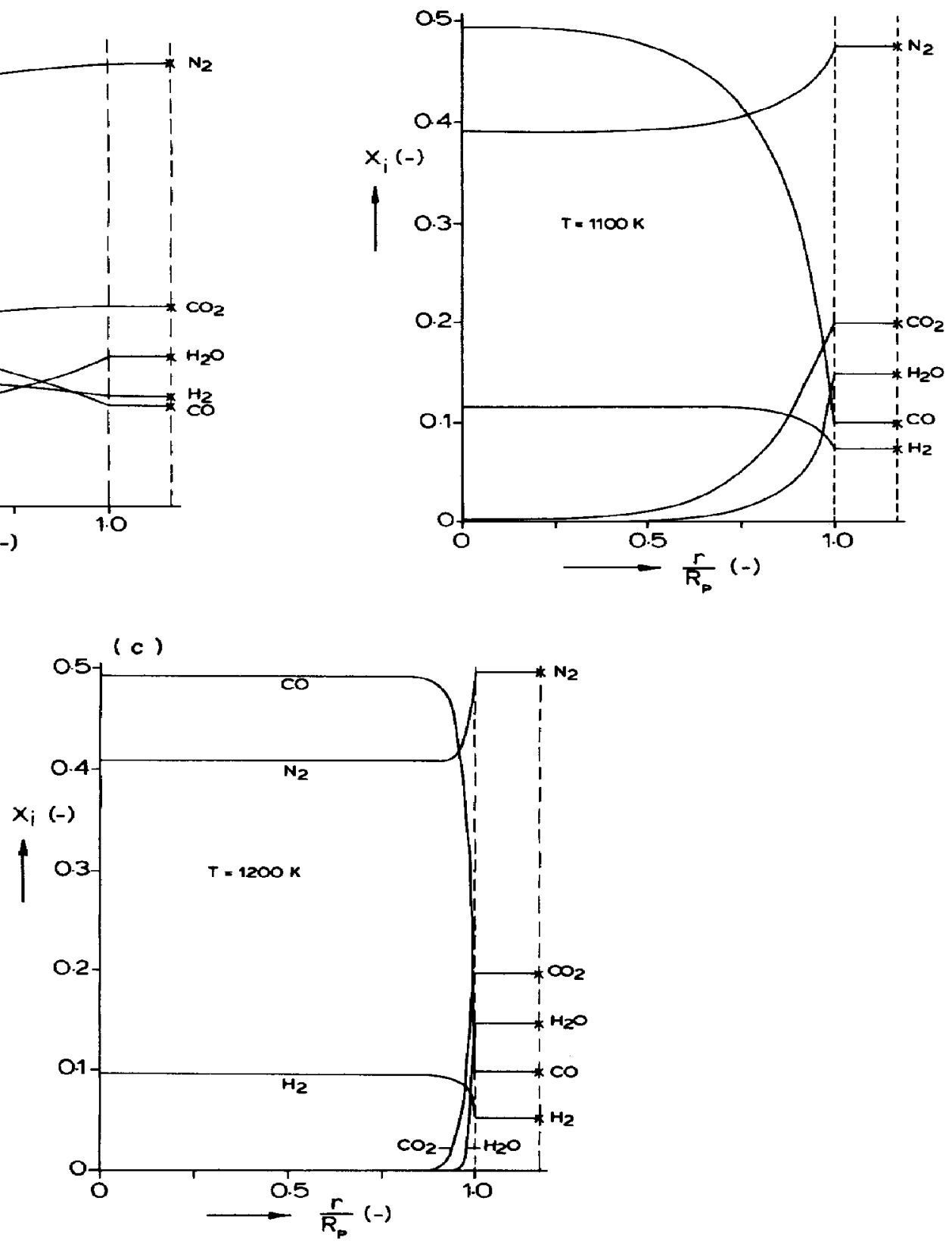

Fig. 2. Intraparticle composition profile for coal $A$ for $X_{c}=0$ and various temperatures. *: Bulk mole fractions. $X_{\mathrm{CO}_{2}}^{b}=0.2, X_{\mathrm{CO}}^{b}=0.1, X_{\mathrm{H}_{2} \mathrm{O}}^{\mathrm{b}}=0.15, S_{h}=10, d_{\mathrm{p}}=0.015 \mathrm{~m}, P^{\mathrm{b}}=0.1013 \mathrm{MPa}$.

here as the ratio of the reaction rate to the reaction rate at bulk conditions:

$$
\eta_{\mathrm{p}}=\frac{\int_{V_{\mathrm{r}}} R_{\mathrm{v}} \mathrm{d} V}{V_{\mathrm{r}}\left[R_{\mathrm{v}}\right]^{\mathrm{b}}}
$$

Despite the fact that, as such, the intraparticle mole fraction gradients for hydrogen are not negligible, taking an infinitely high value for this diffusivity has only a small impact on the particle effectiveness factor $\eta_{p}$, defined by eq. (30). Therefore, this simplifying approach seems to be justified.

The existence of a mole fraction gradient for $\mathrm{ni}$ trogen reflects the fact that gasification reactions are accompanied by a net transfer of momentum out of the particle. The diffusive flux of nitrogen into the particle is balanced exactly by the convective flux out of the particle, as obviously the net flux of nitrogen must be zero. 
1902

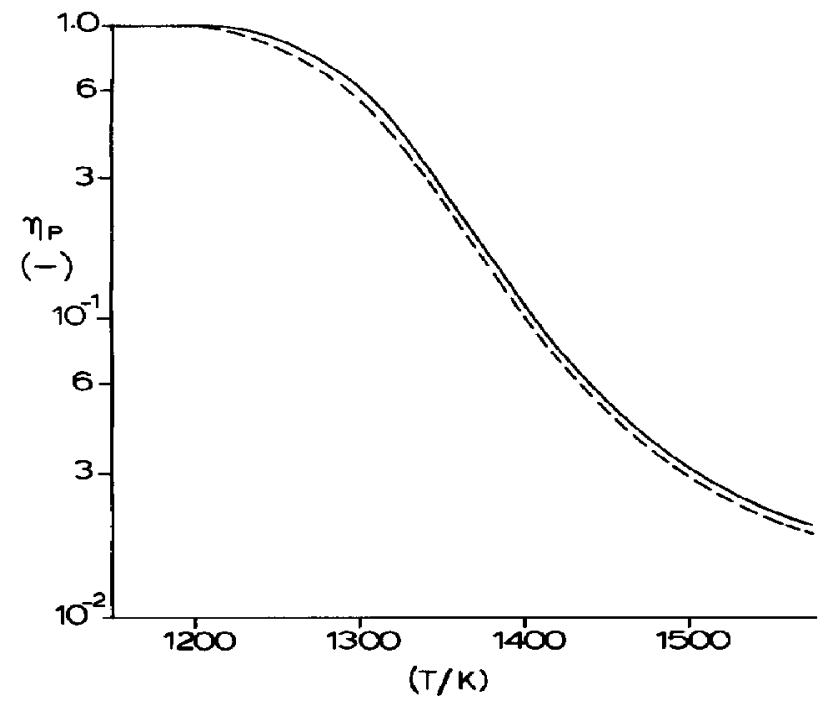

Fig. 3. Particle effectiveness factor vs. temperature for coal A. Conditions as in Fig. 2. - $, \boldsymbol{D}_{\mathbf{H}_{2, i}}=\infty . \cdots, \boldsymbol{D}_{\mathbf{H}_{2}, i}$ according to eq. (11).

For chars with a high porosity, and a correspondingly high value of the diffusive permeability, the steepness of the intraparticle mole fraction gradients is reduced, as is apparent from a comparison of Fig. 2 with Fig. 4. This suggests that the diffusive permeability $k_{o}$ has a considerable impact on the particle effectiveness factor $\eta_{p}$. This impact is shown explicitly in Fig. 5.

The pertinent mass-transfer coefficients in the reaction system studied here are the diffusive permeability $k_{0}$, the viscous permeability $B_{0}$ and the gass film mass-

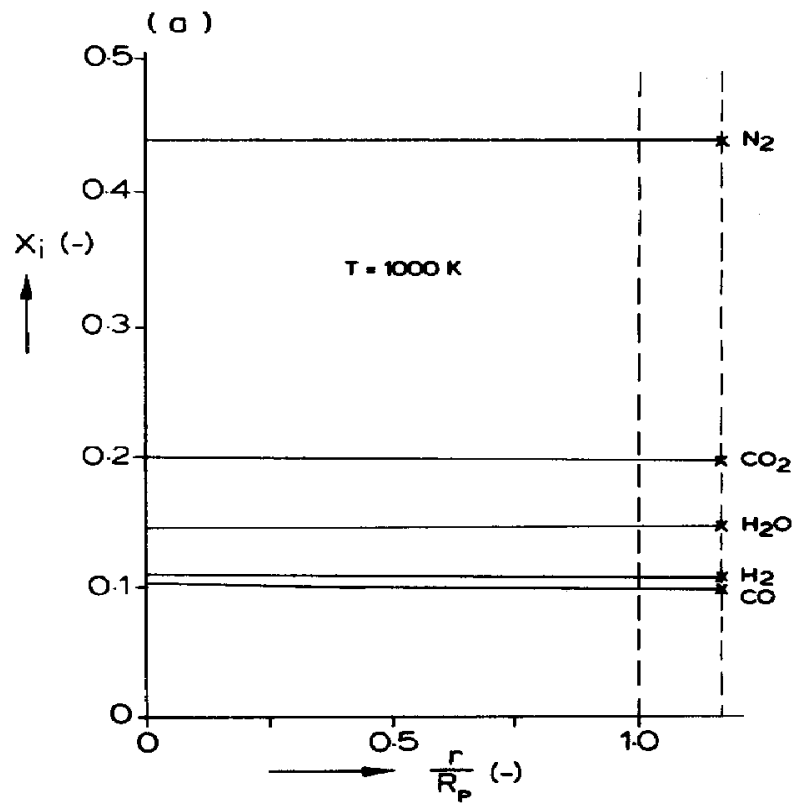

Fig. 4. (a). (b)
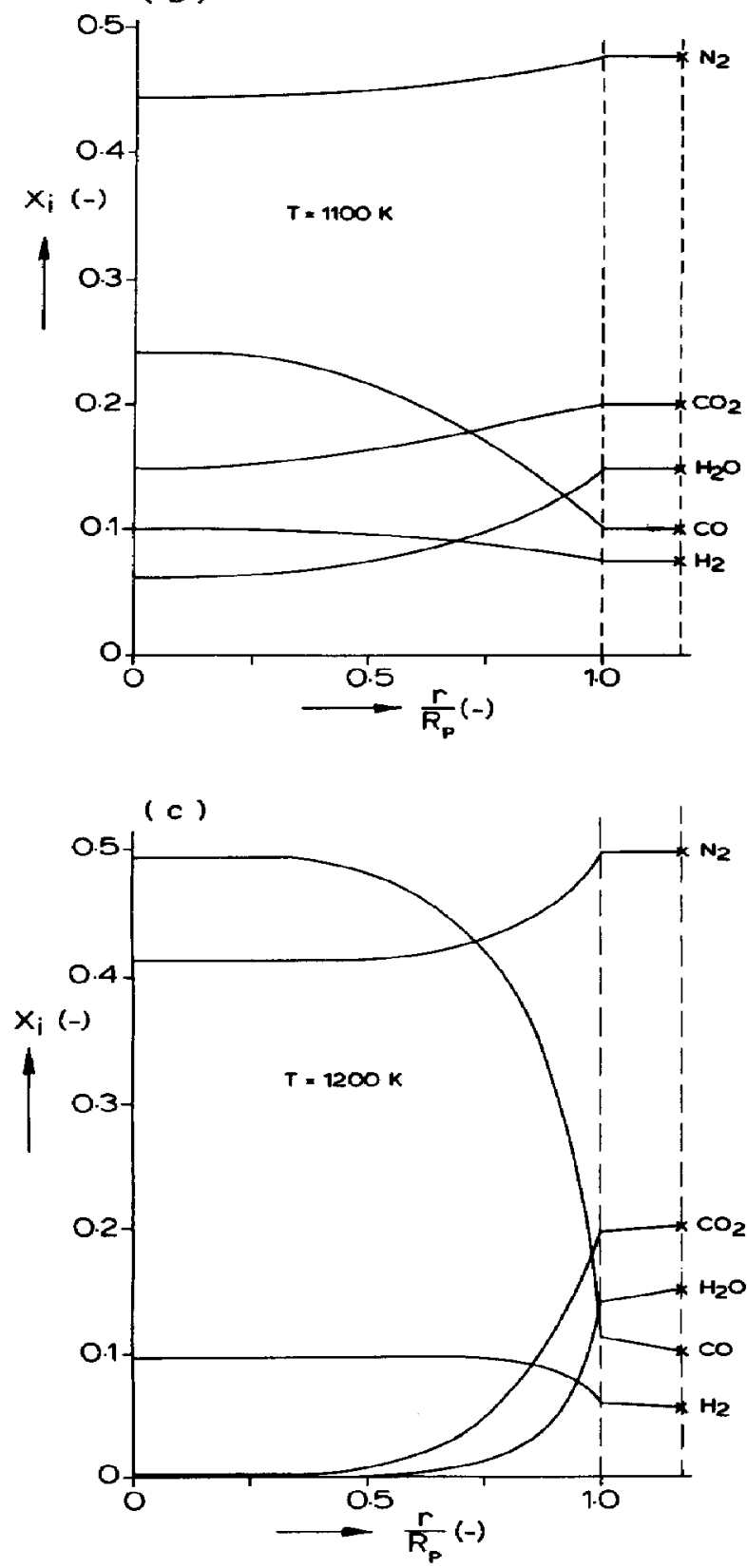

Fig. 4. Intraparticle composition profile for coal A for $X_{c}=0.3$ for $0 \leqslant r \leqslant R_{p}$ and various temperatures. Conditions as in Fig. 2. *: Bulk mole fractions.

transfer coefficient $k_{\mathrm{g} j}$, which is expressed in terms of the Sherwood number. Whereas $k_{\mathrm{o}}$ has a large impact on the overall gasification rate, the influence of $S h$ is small (Fig. 6) and the influence of $B_{0}$ is even totally absent (Fig. 7). Because the intraparticle diffusivities are small as compared to the gas film diffusivity, the bulk gas composition and the gas composition at the gas-solid interphase are nearly equal. Only for $S h<10$ does an effect of the gas film mass transfer become noticeable. Hence the gas film mass-transfer- 


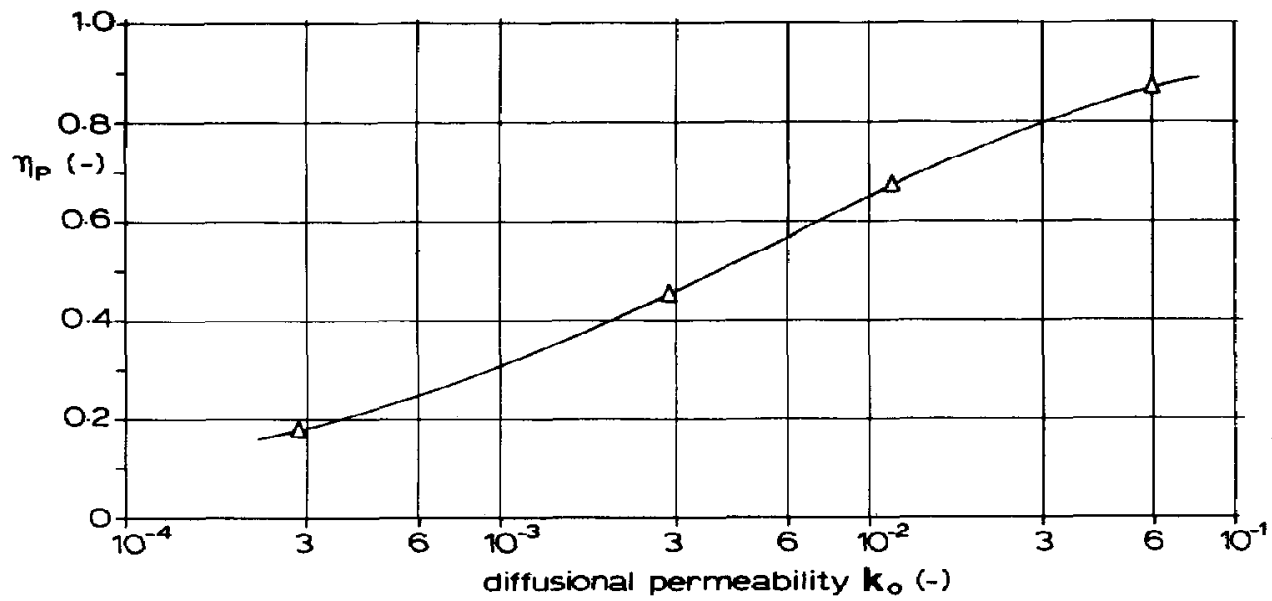

Fig. 5. Particle effectiveness factor vs. the diffusional permeability. Coal $\mathrm{B} ; \mathrm{T}=1050 \mathrm{~K}$; further conditions as in Fig. 2.

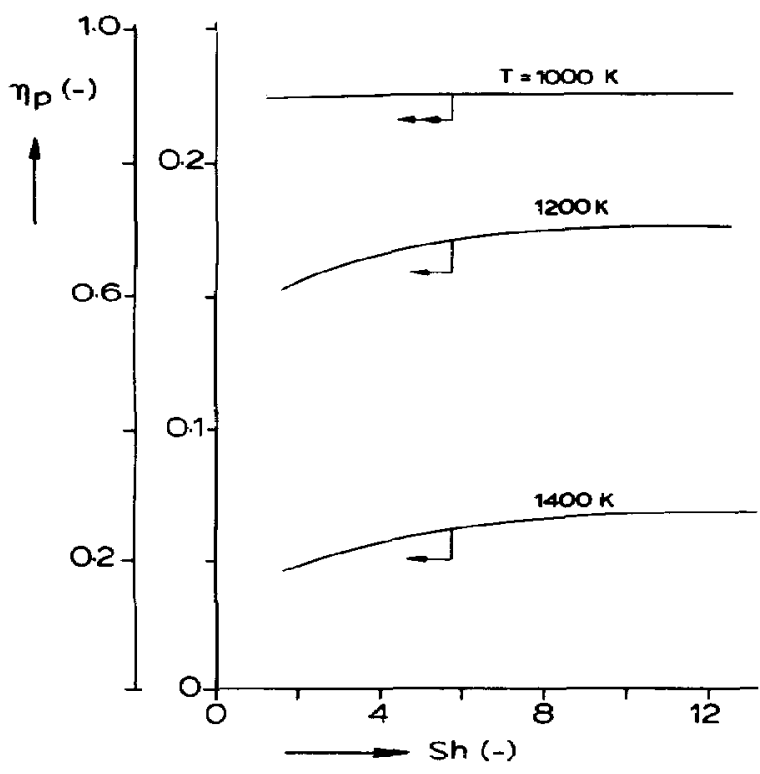

Fig. 6. Particle effectiveness factor vs. the particle Sherwood number for various temperatures. Coal B; conditions as in Fig. 2. controlled regime, the so-called zone 3 , is almost absent. This conclusion was also reached by Desai and Yang [3].

The impact of the viscous permeability $B_{0}$ on the gasification rate is totally absent. This result is not obvious because for low values of $B_{0}$ significant intraparticle pressure gradients develop (Fig. 8). The impact of $B_{0}$ on the effectiveness factor of porous catalyst particles, involved in non-equimolar reactions, has received much attention. On the basis of numerical calculations for binary reaction systems, Abed and Rinker [33] as well as Kehoe and Arris [34] concluded that the particle effectiveness factor is only influenced by $B_{0}$ when diffusion is in the Knudsen or transition regime. From a theoretical analysis of the dusty gas model, Apecetche et al. [35], Williams and Cunningham [36] and Hite and Jackson [37] arrived at the same conclusion, again for binary systems. No theoretical analysis is at present available for multicomponent mixtures. In this work, the dusty gas flux equations were simplified to their continuum limit, and no influence of $B_{0}$ on the particle effectiveness could be observed. This is in agreement with the numerical results presented by Gonzalez et al. [38] for multicom-

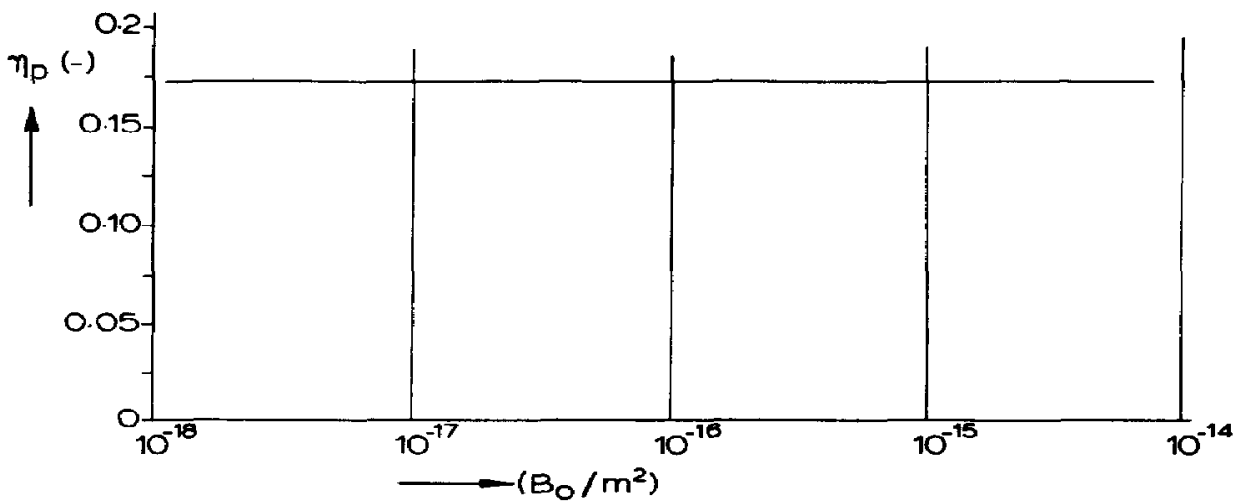

Fig. 7. Particle effectiveness factor vs. the viscous permeability. Coal $\mathrm{A} ; \mathrm{T}=1100 \mathrm{~K}$; further conditions as in Fig. 2. 
ponent flow in the case of catalysed methane reforming. Both results suggest that also in the case of multicomponent mixtures the influence of $B_{0}$ on the particle effectiveness is absent, as long as diffusion is in the continuum regime.

The gasification behaviour of a char particle for various temperatures and particle sizes is indicated in Fig. 9. The impact of temperature is strongest for the low-temperature regime, which is a direct consequence of the strongly non-linear Langmuir-Hinshelwood kinetics. For this reason also, the gasification process shifts rapidly to the chemically controlled regime for low temperatures. Even large particles adopt a homogeneous reaction behaviour at low temperatures, as is indicated in Fig. 10(a). At higher temperature levels the gasification process shifts to a shrinking core reaction behaviour [see Figs 10(b) and 10(c)]. The corresponding conversion-time behaviour is presented in Fig. 11.

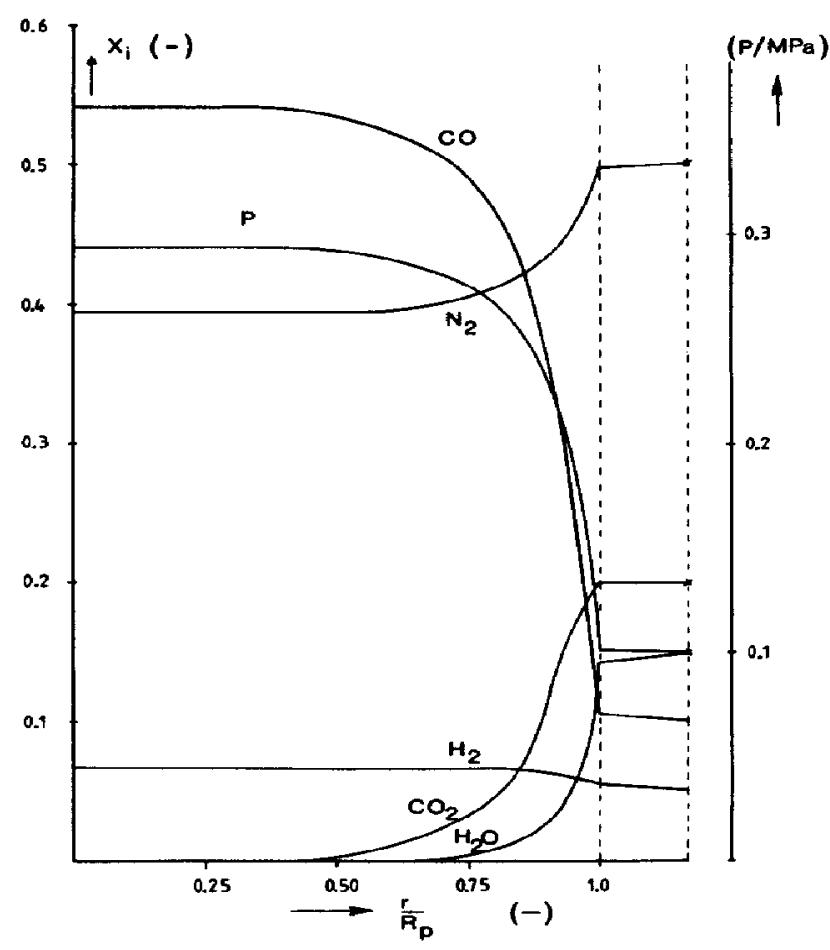

Fig. 8. Intraparticle composition and pressure profile for coal $A$ and a low value of the viscous permeability $B_{0}=5.9 \times 10^{-7} \mathrm{~m}^{2} . T=1200 \mathrm{~K}$; further conditions as in Fig. 2 .

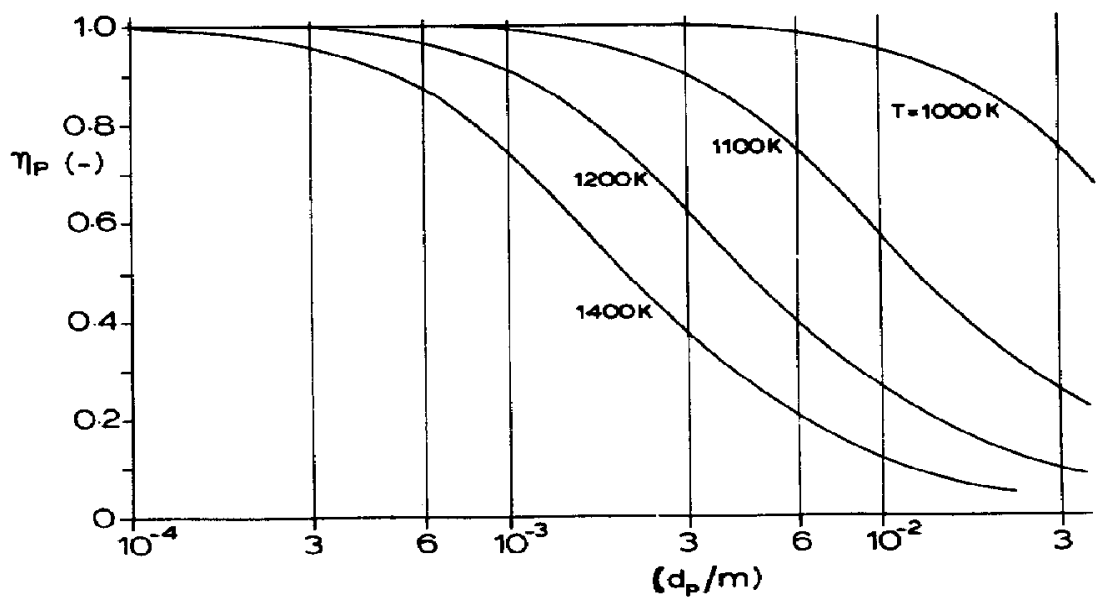

Fig. 9. Particle effectiveness factor vs. the particle size for various temperatures. Coal B; conditions as in Fig. 2. 
(a)

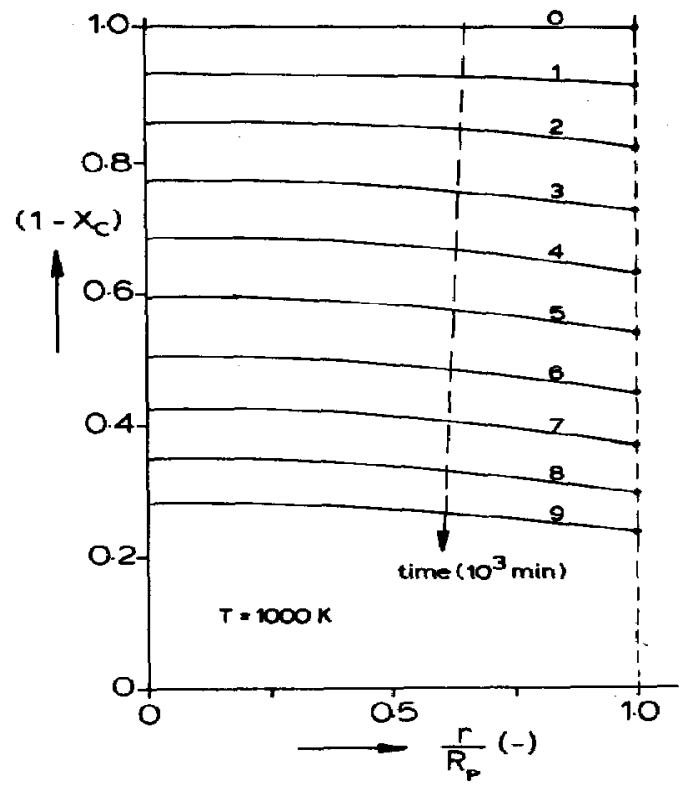

(b)

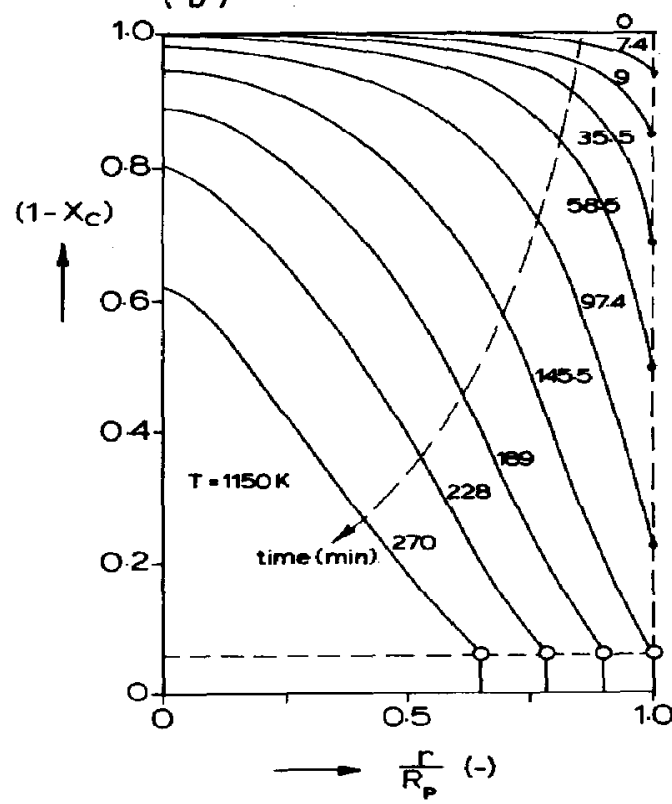

(c)

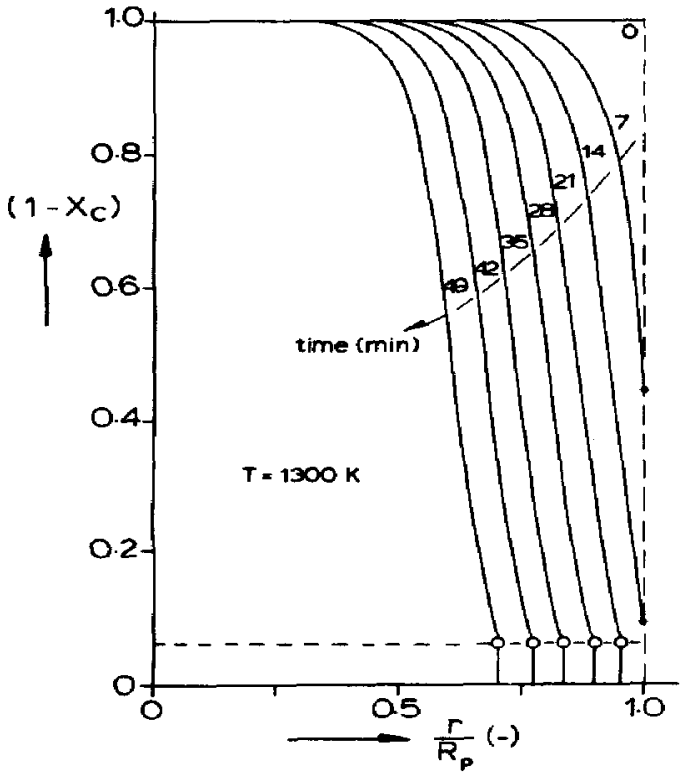

Fig. 10. Intraparticle conversion profile vs. time for various temperatures. Coal B; conditions as in Fig. 2. The dashed line indicates the conversion level where the ash and remaining carbon segregate from the particle.

The conversion rate of a single particle is only moderately affected by the ambient pressure level, as the reaction order of the heterogeneous char gasification reactions is close to zero (Fig. 12).

From the results of the present investigation it can be concluded that the main rate-controlling parameters during the gasification of a single particle are the gasification temperature, the particle size and the diffusive permeability of the particle. In addition, it was shown that macropore diffusion rather than micropore diffusion is rate-controlling.

Because of the strong influence of the porosity on the diffusive permeability, it is essential to incorporate a conversion-dependent diffusivity in simulation models. The influence of the gasification temperature on the overall rate is strong, especially for the lowtemperature regime. This is a result of the non-linearity of the L-H rate equations. Hence, the use of an 


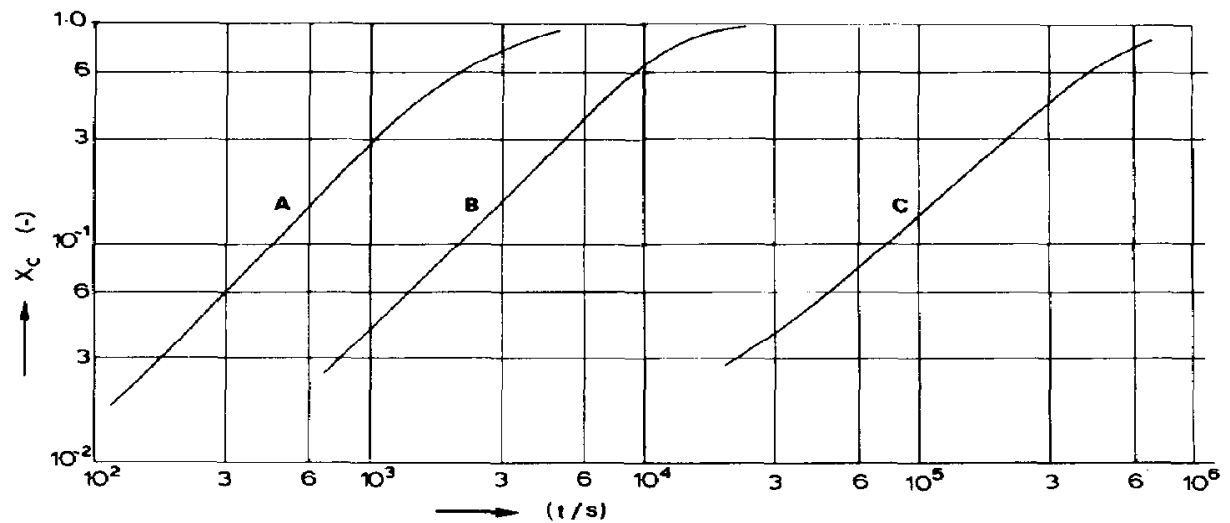

Fig. 11. Conversion-time behaviour for various temperatures. Coal B; conditions as in Fig. 2 . (a) $T$ $=1300 \mathrm{~K}$; (b) $T=1150 \mathrm{~K} ;$ (c) $T=1000 \mathrm{~K}$.

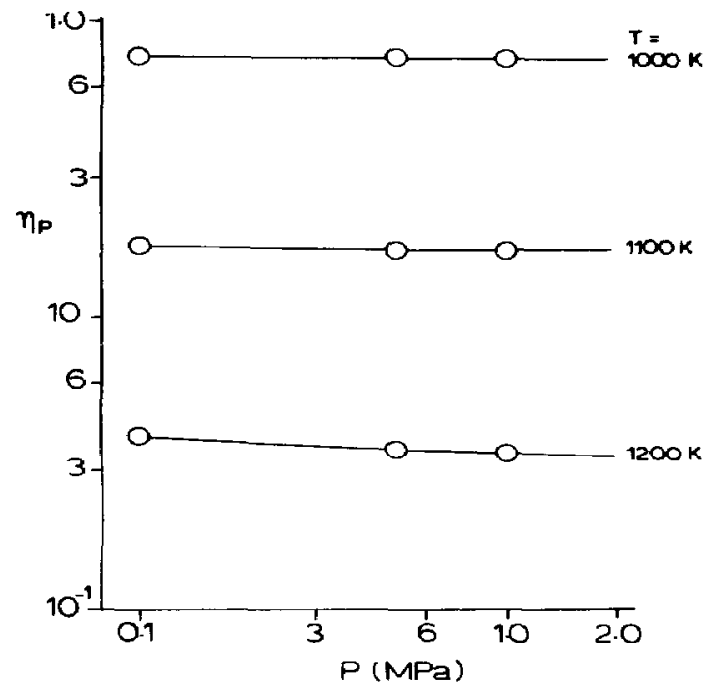

Fig. 12. Particle effectiveness factor vs. the ambient pressure level. Coal A; further conditions as in Fig. 2.

apparant reaction order or the use of an overall pseudo-activation energy is likely to produce erroneous results, in particular at low temperatures. The gasification rate is influenced only to a limited extent by the ambient pressure level and the gas film masstransfer coefficient. The intraparticle flux equations used in the present investigation can be simplified in two ways. First, as long as Knudsen diffusion effects are absent, the use of the dusty gas flux equations is not really justified and they may be replaced by the simpler Stefan-Maxwell equations. Secondly, the diffusivity of hydrogen may be assumed infinitely fast. The error introduced by this simplifying assumption is, in general, quite small.

Acknowledgements - The authors gratefully acknowledge the financial support provided by Shell Coal International Ltd.,
London and The Netherlands Energy Research Foundation, E.C.N. Part of this work was carried out within the framework of the Dutch National Coal Research Programme.

\section{NOTATION}

$\boldsymbol{A}$

$\boldsymbol{B}_{\mathrm{o}}$

$c_{1}$

$C_{i}$

$D_{k, i}$

$D_{i, j}$

$D_{\mathrm{i}, \mathrm{m}}$

$D_{\text {t, }}$

$d_{p}$

$d_{\text {po }}$

$d_{\text {tr }}$

$E_{i}$

$\Delta H_{\mathrm{r}}$

I

$K_{1}, K_{4}$

$K_{2}, K_{3}$,

$K_{\text {s }}$

$K_{5}, K_{6}$

$k_{\text {o }}$

$k_{1}$

$\boldsymbol{k}_{\mathrm{g}, i}$

$k_{\mathrm{g}, i}$

$L_{\text {eff }}$

$M_{i}$

$N_{i}$

n

$P$

R

$\boldsymbol{R}_{\mathrm{v}}$

cross-sectional area, $\mathrm{m}^{2}$

viscous permeability, $\mathrm{m}^{2}$

constant, eq. (41), $\mathrm{m}^{-1}$

concentration, $\mathrm{mol} \mathrm{m}^{-3}$

Knudsen diffusivity, $\mathrm{m}^{2} \mathrm{~s}^{-1}$

binary diffusivity, $\mathrm{m}^{2} \mathrm{~s}^{-1}$

diffusivity in mixture, $\mathrm{m}^{2} \mathrm{~s}^{-1}$

transition regime diffusivity, $\mathrm{m}^{2} \mathrm{~s}^{-1}$

particle size, $\mathbf{m}$

pore size, $\mathbf{m}$

pore mouth size of a pore tree, $\mathrm{m}$ activation energy, $\mathrm{J} \mathrm{mol}^{-1}$

reaction enthalpy, $\mathrm{Imol}^{-1}$

integral, eq. (34), $\mathrm{mol}^{2} \mathrm{~m}^{-5} \mathrm{~s}^{-1}$

intrinsic gasification rate constant, mol $\mathrm{m}^{-2} \mathrm{~Pa}^{-1} \mathrm{~s}^{-1}$

equilibrium constants, $\mathrm{Pa}^{-1}$

homogeneous shift equilibrium constant continuum diffusion permeability Knudsen diffusion permeability, $m$ gas film mass-transfer coefficient for a zero net mole flux at the gas-solid interphase, mol m-2 $\mathrm{s}^{-1}$

gas film mass-transfer coefficient with non-zero net mole flux at the gas-solid interphase, $\operatorname{mol~m} \mathrm{m}^{-2} \mathrm{~s}^{-1}$

effective pore length, $m$ molecular mass, $\mathbf{k g ~ m o l}^{-1}$

mole flux relative to a fixed coordinate system, mol m-2 $\mathrm{s}^{-1}$

number of components in mixture

pressure, $\mathbf{P a}$

gas constant, $\mathrm{J}^{\mathrm{mol}^{-1}} \mathrm{~K}^{-1}$

volumetric char conversion rate, mol $\mathbf{m}^{-3} \mathrm{~s}^{-1}$ 


\begin{tabular}{|c|c|}
\hline $\boldsymbol{R}_{\mathbf{i}}$ & $\begin{array}{l}\text { intrinsic char conversion rate, mol } \\
m^{-2} s^{-1}\end{array}$ \\
\hline $\boldsymbol{R}_{\mathrm{p}}$ & particle radius, m \\
\hline$r$ & radial distance from particle centre, $\mathbf{m}$ \\
\hline $\begin{array}{l}S_{\mathrm{p}} \\
S h\end{array}$ & $\begin{array}{l}\text { specific surface area, } \mathrm{m}^{2} \mathrm{~g}^{-1} \\
\text { particle Sherwood number, see eq. (28) }\end{array}$ \\
\hline $\boldsymbol{t}$ & time, $s$ \\
\hline$T$ & temperature, $\mathbf{K}$ \\
\hline$V_{\mathrm{p}}$ & particle volume, $\mathbf{m}^{3}$ \\
\hline$V_{r}$ & reaction volume, $\mathbf{m}^{3}$ \\
\hline$X_{i}$ & mole fraction \\
\hline $\boldsymbol{X}_{\mathrm{c}}$ & char conversion \\
\hline
\end{tabular}

\section{Greek letters}

$\boldsymbol{\beta}$

$\psi$

$\phi_{\mathrm{p}}$

$\eta_{\mathrm{p}}$

$\mu_{m}$

$\varepsilon_{p}$

$\gamma$

$\tau$

$\lambda$

$\boldsymbol{\rho}$

$\boldsymbol{\Omega}_{\mathrm{d}}$

$\sigma_{i, j}$

Subscripts
$\boldsymbol{i}$
$\mathbf{s}$
$\mathbf{m}$
$\mathbf{p}$
$\mathbf{o}$

refers to ith species

refers to solid

refers to gas mixture

refers to particle

refers to initial conditions

\section{Superscripts}

$\begin{array}{ll}\text { e } & \text { effective value } \\ \text { b } & \text { value at the bulk gas phase } \\ \text { s } & \text { value at the gas-solid interphase } \\ \text { value at the pore mouth }\end{array}$

\section{REFERENCES}

[1] Bliek A., Mathematical modeling of a cocurrent fixed bed coal gasifier. Ph.D. thesis, Twente University of Technology, The Netherlands 1984.

[2] Srinivas B. and Amundson N. R., A single-particle char gasification model. A.I.Ch. E. J. 198026487.

[3] Desai N. J. and Yang R. T., Kinetics of hightemperature carbon gasification reaction. A.I.Ch.E. J. 198228237.

[4] Jackson R., Transport in Porous Catalyst. Elsevier, Amsterdam 1977.

[5] Simons G. A. and Finson M. L., The structure of coal char: p.1. Pore branching. Combust. Sci. Technol. 1979 19217.

[6] Gavalas G. R., A random capillary model with application to char gasification at chemically controlled rates. A.I.Ch.E. J. 198026577.

[7] Hashimoto K. and Silveston P. L., Gasification-pI:
Isothermal, kinetic control model for a solid with a pore size distribution. pII: Extension to diffusion control. A.I.Ch.E. J. 197319259.

[8] Wen $C$. $Y$. and $W u N$. T., An analysis of slow reactions in a porous particle. A.I.Ch.E. J. 1976221012.

[9] Froment G. F. and Bischoff K. B., Chemical Reactor Analysis and Design, Chap. 3.6. Wiley, New York 1979.

[10] Yang R. T. and Steinberg $M$., A diffusion cell method for studying heterogencous kinetics in the chemical reaction/diffusion controlled regime. Kinetics of $\mathrm{C}$ $+\mathrm{CO}_{2} \rightarrow 2 \mathrm{CO}$ at $1200-1600^{\circ} \mathrm{C}$. Ind. Engng Chem. Fundam. 197716235.

[11] Arri L. E. and Amundson N. R., An analytical study of single particle char gasification. A.I.Ch.E. J. 19782472.

[12] Mason E. A., Malinauskas A. P. and Evans R. B., Flow and diffusion of gases in porous media. J. Chem. Phys. 1967463199.

[13] Fredersdorff C. G. Von and Elliott M. A., Chemistry of Coal Utilization (Edited by Lowry H. H.). Wiley, New York 1963.

[14] Bhatia S. K. and Perlmutter D. D., A random pore model for fluid solid reactions: I. Isothermal, kinetic control. A.I.Ch.E. J. 198026379.

[15] Gan H., Nandi S. P. and Walker P. L.. The nature of the porosity in American coals. Fuel 197251272.

[16] Wakao $N$. and Smith J. M., Diffusion in catalyst pellets. Chem. Engng. Sci. 196217825.

[17] Hite R. H. and Jackson R., Pressure gradients in Porous catalyst pellets in the intermediate diffusion regime. Chem. Engng Sci. 197732703.

[18] Deriaguin B. V. and Bakanov S. P., Theory of gas flow in a porous body near the Knudsen regime. Soviet Phys.-Dokl. 19572326.

[19] Evans R. B., Truitt J. and Watson G. M., Interdiffusion of helium and argon in a large-pore graphite. $J$. chem. Engng Data 19616522.

[20] Evans R. B., Watson G. $M$. and Mason E. A., Gaseous diffusion in porous media at uniform pressure. J.Chem. Phys. 1961352076.

[21] Rothfeld L. B., Gaseous counterdiffusion in catalyst pellets. A.I.Ch.E. J. 1963919.

[22] Scott D. S. and Dullien F. A. L., Diffusion of ideal gases in capillaries and porous solids. A.I.Ch.E. J. 19628113.

[23] Yang R. T. and Liu R. T., Gaseous diffusion in porous solids at elevated temperatures. Ind. Eng. Chem. Fundam. 198221262.

[24] Klavetter E. A., Liapis A. I., Crossner O. K. and Litchfield R. J., Comparison of mass fuxes predicted by the dusty-gas and a modified dusty-gas model. Chem. Engng Sci. 198237997.

[25] Chen O. T. and Rinker R. G., Modification of the dustygas equation to predict mass transfer in general porous media. Chem. Engng Sci. 19793451.

[26] Feng C., Kostrov V. V. and Stewart W. E.; Multicomponent diffusion of gases in porous solids. Models and experiments. Ind. Engng Chem. Fundam. 1974135.

[27] Gavalas G. R. and Wilks K. A., Intraparticle mass transfer in coal pyrolysis. A.I.Ch.E. J. 198026201.

[28] Russel W. B., Saville D. A. and Greene, M. I., A model for short residence time hydropyrolysis of single coal particles. A.I.Ch.E. J. 19792565.

[29] Dutta S., Wen C. Y. and Belt R. J., Reactivity of coal and char 1 in carbon dioxide atmosphere. Ind. Engng Chem. Proc. Des. Dev. 19771620.

[30] Prater C. D., The temperature produced by heat of reaction in the interior of porous particles. Chem. Engng Sci. 19588284.

[31] Bird R. B., Stewart W. E. and Lightfoot E. N., Transport Phenomena, Chap. 21. Wiley, New York 1960.

[32] Wilke C. R., Diffusional properties of multicomponent gases. Chem. Engng Progr. 19504695.

[33] Abed R. and Rinker R. G., Reaction with mole changes in porous catalysts in the molecular transition, and knudsen regimes. A.I.Ch.E. J. 1973196. 
[34] Kehoe J. P. G. and Aris R., Communications on the theory of diffusion and reaction - IX. Internal pressure and forced flow for reactions with volume change. Chem. Engng Sci. 1973282094.

[35] A pecetche M. A., Gonzalez M. G., Williams R. J. J. and Cunningham R. E., Viscous and diffusive transport with simultaneous chemical reaction in non-isobaric porous catalyst particles. J. Catalysis 197329451 .

[36] Williams R. J. J. and Cunningham R. E., Pressure gradients in porous catalyst pellets. Chem. Engng Sci. 197934157.

[37] Hite R. H. and Jackson R., Pressure gradients in porous catalyst pellets. Chem. Engng Sci. 197934158.

[38] Gonzalez M. G., Laborde M. A. and Williams R. J. J., Influence of the different permeabilities of a catalyst pellet on its effectiveness. Ind. Engng Chem. Proc. Des. Dev. 198019498.

[39] Abed R., Ha K. and Rinker R. G., nth order reaction with mole changes in porous catalysts in the molecular, transition and knudsen regimes. A.I.Ch.E. J. 197420 391.

[40] Feng C. and Stewart W. E., Practical models for isothermal diffusion and flow of gases in porous solids. Ind. Engng Chem. Fundam. 197312143.

[41] Gunn $R$. D. and $K$ ing $C$. $J$., Mass transport in porous media under combined gradients of composition and pressure. A.I.Ch.E. J. 196915507.

[42] Mason E. A. and Malinauskas A. P., Gas Transport in Porous Media: The Dusty Gas Model, Chem. Engng Monograph 17. Elsevier, Amsterdam 1983.

[43] Nandi S. P., Ramadass V. and Walker P. L., Changes in the ultrafine structure of anthracites upon heat treatment. Carbon 19642199.

[44] Otani S., Wakao N. and Smith J. M., pII: Diffusion and flow in porous catalysts. A.I.Ch.E. J. 196511 439. pIII: Effect of pressure gradients on the effectiveness of porous catalysts. A.I.Ch.E. J. 196511446.

[45] Petersen E. E., Reaction of porous solids. A.I.Ch.E. J. 19573443.

[46] Roy T. D. and Abraham K. P., An analysis of the pressure build-up inside a reacting pellet during gas-solid reactions. Metall. Trans. 19745349.

[47] Simons G. A., Char gasification: p.1. Transport model. Combust. Sci. Technol. 197920107.

[48] Walker P. L., Rusinko F. and Austin L. G., Gas reactions of carbon. Adv. Catalysis 195911133.

[49] Wong R. L. and Denny V. E., Diffusion, flow and heterogeneous reaction of ternary mixtures in porous catalytic media. Chem. Engng Sci. 197530709.
APPENDIX A. MICROPORE DIFFUSION EFFECTS

In order to assess the validity of the "smooth-field" assumption, which states that the reaction conditions are the same for all pores located at a given macroscopic position, the effects of micropore diffusion have to be assessed. Micropore effectivity factors can be evaluated on the basis of the generalized Thiele modulus $\phi[9]$ :

$$
\phi=R_{\mathrm{v}}^{\bullet} \frac{V}{A} \frac{1}{\sqrt{2}}\left[\int_{0}^{C \bullet} D R_{\mathrm{v}} \mathrm{d} C\right]^{-1 / 2} .
$$

When the expression for the Thiele modulus is applied to a single pore or to a pore tree, the superscript - denotes the value at the pore mouth. The reaction rate per unit of pore volume, $\boldsymbol{R}_{\mathrm{v}}$, can be converted into the intrinsic reaction rate per unit of pore wall surface area:

$$
\boldsymbol{R}_{\mathbf{v}}=\frac{S}{V} \boldsymbol{R}_{\mathbf{i}}
$$

In the case of the carbon- $\mathrm{CO}_{2}$ reaction, the in trinsic reactivity $R_{i}$ is given by eq. (1). If $D$ is taken to be invariant with the gas composition, eq. (31) can be simplified to:

$$
\phi=R_{\mathrm{i}}{ }^{D^{-1 / 2}} \frac{\sqrt{(V S)}}{A} \frac{1}{\sqrt{2}} I^{-1 / 2}
$$

with:

$$
I=\int_{0}^{P C O_{2}^{\circ}} \frac{1}{R T}\left\{\frac{K_{1} P C O_{2}}{1+K_{2} P C O_{2}+K_{3} P C O}\right\} \mathrm{dPCO}_{2}
$$

The pore volume $V$, the pore cross-sectional area $A$ and the pore wall surface arca $S$ can be expressed as:

$$
\begin{aligned}
& V=\frac{\pi}{4} d_{\mathrm{po}}^{2} L_{\mathrm{eff}} \\
& S=\pi d_{\mathrm{po}} L_{\mathrm{eff}} \\
& A=\frac{\pi}{4} d_{\mathrm{po}}^{2}
\end{aligned}
$$

Equation (34) can be solved only when a relation between $\mathrm{PCO}_{2}$ and $\mathrm{PCO}$ is found. When bulk flow in the micropore regime is neglected, and when equal binary diffusivities are assumed for $\mathrm{CO}$ and $\mathrm{CO}_{2}$, the reaction stoichiometry requires:

$$
\mathrm{PCO}^{-}-\mathrm{PCO}=2\left[\mathrm{PCO}_{2}-\mathrm{PCO}_{2}^{\bullet}\right]
$$

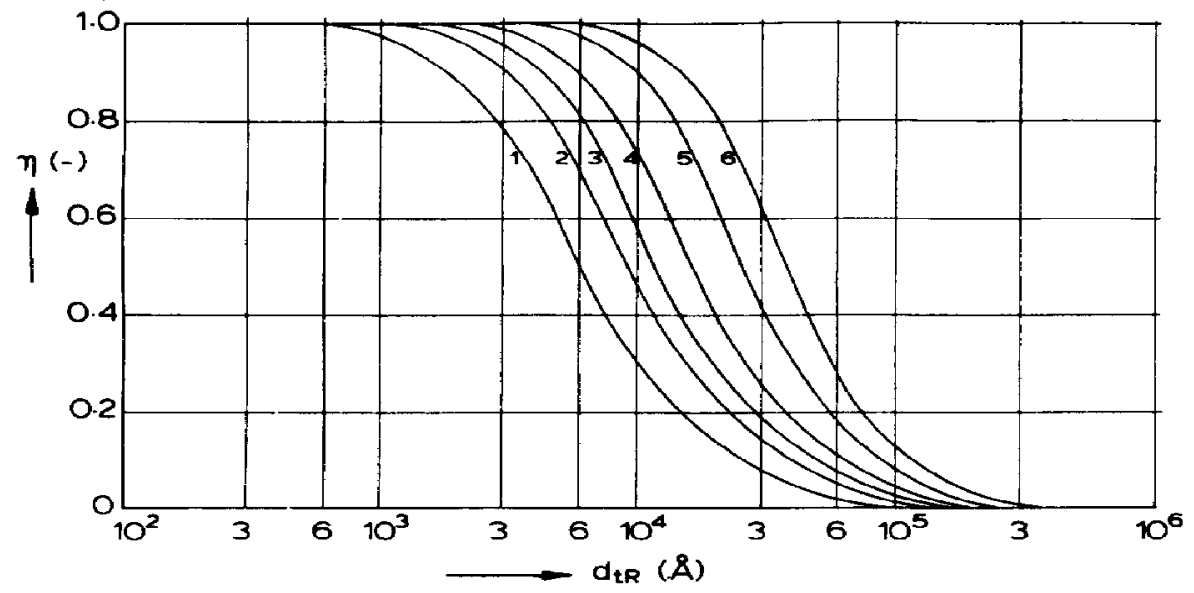

Fig. 13. Pore tree effectiveness factor vs. the trunk size $d_{\mathrm{tr}}$ (see Fig. 1). Coal A; $X_{\mathrm{CO}}=X_{\mathrm{CO}}=0.2 ; P b$ $=0.1013 \mathrm{MPa} ;(1)-(6): T=1773,1673,1573,1473,1373$ and $1273 \mathrm{~K}$. 
Using eqs (35)-(38), the pore Thiele modulus can be expressed as:

$$
\phi=R_{\mathrm{i}} D^{-1 / 2} L_{\mathrm{eff}} d_{\mathrm{po}}^{-1 / 2} \frac{2}{\sqrt{2}} I^{-1 / 2}
$$

with:

$$
\begin{aligned}
I= & \frac{K_{1}}{R T} \frac{1}{K_{2}-2 K_{3}}\left[\mathrm{PCO}_{2}^{\circ}+\left(\frac{1+K_{3} P C O^{\bullet}+2 K_{3} P C O_{2}^{\bullet}}{K_{2}-2 K_{3}}\right)\right. \\
& \left.\times \ln \left(\frac{1+2 K_{3} P C O_{2}^{\ominus}+K_{3} P C^{\bullet}}{1+K_{3} P^{\bullet}+K_{2} P C O_{2}^{\bullet}}\right)\right]
\end{aligned}
$$

For a single pore the effective pore length equals the true pore length, and eq. (39) can be solved immediately. If the micropore system consists of pore branches, as suggested by the pore tree model of Simons and Finson [5], the effective length of a single pore is increased because many smaller pores radiate from it. According to the analysis of Simons and Finson, the effective length of a pore tree with a pore mouth $d_{\mathrm{tr}}$ (see Fig. 1) scales as $d_{\mathrm{tr}}^{2}$ :

$$
L_{\mathrm{eff}}=c_{1} d_{\mathrm{tr}}^{2}
$$

Equations (41) and (39) suggest that the pore tree Thiele modulus increases with increasing pore mouth sizes. Therefore large pore trees tend to be diffusion-controlled. Although Gavalas [6] used a random pore model instead of a tree model, he arrived at the same conclusion.

The maximum size of a pore tree is governed by constraints with regard to the total pore volume and the total internal surface area of a particle. On the basis of the model equations presented by Simons and Finson [5], the maximum size of $d_{t r}$ is estimated to be $0.2-0.4 \mu \mathrm{m}$, with $c_{1}=8 \times 10^{9} \mathrm{~m}^{-1}$.

Using eq. (18) for the diffusivity $D$ and eq. (41) for the effective length of a pore tree system, the pore tree Thiele modulus can be assessed for varying values of $d_{\mathrm{tr}}$. The pore tree effectiveness factor is given by:

$$
\eta_{\mathrm{tr}}=\frac{\tanh (\phi)}{\phi}
$$

The results for the anthracite char presently investigated are presented in Fig. 13. When it is kept in mind that the trunk size $d_{\mathrm{tr}}$ is limited to $0.4 \mu \mathrm{m}$, the results indicate that the micropore effectiveness factor exceeds 0.9 for temperatures up to $1400^{\circ} \mathrm{C}$. Therefore the smooth-field assumption applies up to this temperature level for the conditions given in Fig. 13. 